\title{
Sonification of Population Behavior in Particle Swarm Optimization
}

\author{
Tiago F. Tavares \\ University of Campinas \\ Laboratory of Signal Processing for \\ Communications \\ School of Electrical and Computer Engineering \\ Av. Albert Einstein, 400 \\ Campinas, SP, Brazil \\ tavares@dca.fee.unicamp.br
}

\author{
Alan Godoy \\ University of Campinas \\ Laboratory of Bioinformatics and Bioinspired \\ Computing \\ School of Electrical and Computer Engineering \\ Av. Albert Einstein, 400 \\ Campinas, SP, Brazil \\ godoy@dca.fee.unicamp.br
}

\begin{abstract}
High-dimensional optimization problems may be addressed using populational meta-heuristics, whose statistical properties may indicate important characteristics of the optimization process. There is a great number of these properties, which means their joint visualization may become impractical. We developed a method for sonically displaying characteristics of population dynamics in particle swarm optimization processes as soundscape parameters. This process allows jointly analyzing several dimensions of the population's dynamics. Moreover, design decisions aimed at generating aesthetically appealing soundscapes, which allows the proposed system to be used as an automated music composition environment.
\end{abstract}

\section{INTRODUCTION}

Sonification is the process of displaying useful information through a non-speech, sonic interface so that its behavior can be more accurately informed to a human user [3]. There are many practical uses for sonification, one of which is sonically displaying data for exploration [2].

We propose a system for the sonification of the population dynamics in Particle Swarm Optimization (PSO), a system with multiple interacting agents aimed at locating the best solution for a real-parameter problem. As PSO's optimization ability is the product of the swarm's collective behavior, it is interesting to develop ways to efficiently monitor the population. Our system allows jointly analyzing relevant characteristics of agent populations by mapping them into soundscape parameters. The final sonic results aim at giving insight regarding the general behavior of the optimization algorithm. In addition, the soundscapes were designed to be aesthetically appealing, which means that they can be listened to as artistic musical pieces generated by an algorithmic composition process. Although populational

Permission to make digital or hard copies of all or part of this work for personal or classroom use is granted without fee provided that copies are not made or distributed for profit or commercial advantage and that copies bear this notice and the full citation on the first page. To copy otherwise, to republish, to post on servers or to redistribute to lists, requires prior specific permission and/or a fee.

GECCO'13, July 6-10, 2013, Amsterdam, The Netherlands.

Copyright 2013 ACM TBA ...\$15.00. meta-heuristics have been largely used to compose music, for example in work by Blackwell and Young [1] and by Jones [4], to our knowledge no composition process used a mapping of the characteristics of the population dynamics instead of their results - for the generation of soundscapes. The proposed system is closer to the well-known fractal image generators, which may be viewed as an equation display, but also simply as artistic products.

\section{PARTICLE SWARM OPTIMIZATION}

The Particle Swarm Optimization (PSO) [5] is a populational meta-heuristic for optimization of real-valued search spaces problems. Unlike most Evolutionary Algorithms, which draw inspiration from genetics and Darwinian evolution, Particle Swarm Optimization, as its name suggests, is based on collective behavior of social animals and insects, specially on social sharing of information in search and retrieval of food. In PSO, each individual (known as particle in this context) moves through the search space with its own velocity, evaluating the quality of each potential solution visited. The particle, then, combine its velocity with information about its own history (cognitive influence) and the history of its social contacts (social influence) to define the next direction to explore. Some parameters control how information is exchanged between the particles and how these informations are combined. The inertia parameter controls the influence of the previous velocity on the search direction, while the cognitive and the social parameters define the weight each individual will give, respectively, to its own history and to influence from its peers. The network of social contacts (known as "neighborhood") is also an important parameter that defines much of the collective behavior of the swarm: highly connected neighborhoods stimulate a faster convergence, which may trap the swarm in a low quality local optima, whereas if the connections are too sparse convergence may be delayed, allowing the swarm to spend much time in non-promising regions when it could be better to focus on improving the best solution found so far [6].

\section{THE SONIFICATION PROCESS}

Many measures were developed to analyze the behavior of a swarm, being used to assist in the process of fine-tuning parameters for a problem and also to analyze the reasons for the success or failure of a swarm in locating good solutions. Examples of these measures are the average velocity of the 


\begin{tabular}{|c|c|c|}
\hline Population characteristic & Key concept & Sonic parameter \\
\hline $\begin{array}{c}\text { Greater speed of the swarm in the search space } \\
\text { Greater population alignment } \\
\text { Greater diversity } \\
\text { Greater relative fitness gain } \\
\text { Population is further away from the best solution so far } \\
\text { Population is closer to the global optimum }\end{array}$ & $\begin{array}{l}\text { Speed } \\
\text { Simplicity } \\
\text { Ambience } \\
\text { Energy } \\
\text { Tension } \\
\text { Serenity }\end{array}$ & $\begin{array}{c}\text { Notes are yielded faster } \\
\text { Less harmonics are produced } \\
\text { More echo is heard } \\
\text { Increased loudness } \\
\text { Notes have greater chance of reaching higher pitches } \\
\text { Notes have greater duration }\end{array}$ \\
\hline
\end{tabular}

Table 1: Metaphors used in sonification.

particles in a swarm, the alignment between these particles, the diversity of positions currently being explored, the fitness evolution and the distances from the centroid of the swarm to the best solution found so far and to the global optimum (a measure only available for functions in which the location of the global optimum is known). Each measure will provide only a glimpse into the overall behavior of the swarm, but by monitoring a set of metrics throughout the search one can get valuable insights about the system's inner workings and the necessary adjustments. Real-time monitoring of multiple metrics, however, can be very difficult, hence it is necessary to look for a new way to display informations about the behavior of the swarm. To solve this problem, we propose synthesizing a soundscape whose parameters are directly controlled by the relevant measures that describe the population behavior.

The sound generation system is based on a monophonic note synthesis module that, when requested by a controller, yields musical notes that are characterized by its magnitude, duration, pitch and timbre. We employed a parametermapping-based sonification. This mapping was constructed by selecting a key concept that best describes it in the context of a multi-agent optimization process, and then selecting a sonic parameter that reflects that same concept. The result of this process is shown in Table 1.

Since all characteristics of the dynamics are being displayed at the same time, a great magnitude in one (or a few) of them can mask important differences in the others. Preliminary tests have shown that this is specially true when search spaces of different sizes and dimensions are being compared - in this case, the consequent difference in the swarm speed (related to the sound tempo) masks the other relevant differences. Hence, it may be helpful to normalize the population speed and all distance-based features of each dynamic before sonifying it.

\section{TESTS AND RESULTS}

The evaluation process consisted of a careful analysis of the generated soundscapes, aiming at detecting if meaningful representations of the multi-agent dynamics could be produced. We used a Particle Swarm Optimization process to produce the sonification coefficients, and we evaluated changes on the swarm size, the function to be optimized, the swarm parameters (social and individual) and the swarm neighborhood. All sound examples used in this section are available for immediate listening at the URL http:

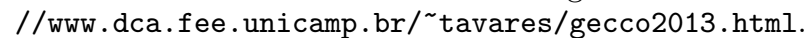

Overall, a correlation between the sonification results and the swarm behaviour can be observed when comparing the generated soundscapes with visual data. Especially interesting auditory artifacts are generated when a new attractor is found. Also, the generated soundscapes clearly end with a slow rhythm that depicts the end of the optimization process.

\section{CONCLUSION}

This paper described a method for the sonification of the behavior of a population in PSO dynamics, sonically displaying relevant features of these dynamics. It was noted that long-term measure variations are harder to observe, but short-term variations are more noticeable. Also, the generated soundscapes are useful for generating insight on how the population behaves, which may lead to education and research applications.

The proposed method was tested using Particle Swarm Optimization, but any populational heuristics (Genetic Algorithms or Ant Colony Optimization, for example), or even other variations of PSO could be immediately used. This would require using a set of measures that are more meaningful for the specific type of dynamics that would be sonified. Hence, this points a possible direction for future work.

\section{REFERENCES}

[1] T. Blackwell and M. Young. Self-organised music. Org. Sound, 9(2):123-136, Aug. 2004.

[2] A. de Campo, C. Frauenberger, and R. Holdrich. Designing a generalized sonification environment. In Proceedings of ICAD 04-Tenth Meeting of the International Conference on Auditory Display, Sydney, Australia, July 2004.

[3] T. Hermann, A. Hunt, and J. G. Neuhoff. The Sonification Handbook. Logos Publishing House, Berlin, 1 edition, 2011.

[4] D. Jones. Atomswarm: A framework for swarm improvisation. In EvoWorkshops, 2008.

[5] J. Kennedy and R. C. Eberhart. Particle swarm optimization. In Proceedings of IEEE International Conference on Neural Networks, volume 4, pages 1942-1948, Dec. 1995.

[6] J. Kennedy and R. Mendes. Population structure and particle swarm performance. In Proceedings of the 2002 Congress on Evolutionary Computation, 2002. CEC 'O2, volume 2, pages 1671-1676, 2002. 